\title{
Here, there, but not everywhere: Adoption and diffusion of IoT in Swedish municipalities
}

\author{
Ted Saarikko \\ Umeå University \\ ted.saarikko@umu.se
}

\author{
Ulrika H. Westergren \\ Umeå University \\ ulrika.westergren@umu.se
}

\author{
Katrin Jonsson \\ Umeå University \\ katrin.jonsson@umu.se
}

\begin{abstract}
The Internet of Things (IoT) brings with it promises of smart cities with improved efficiency, increased transparency, and enhanced public services. However, few studies have empirically and systematically investigated the reasoning behind the decision to adopt IoT within municipal organizations. In this paper we study the adoption and diffusion of IoT in Swedish municipalities. We outline areas of application and perceived value creation and conclude that the main reasons for adoption and diffusion can be traced back to 1) the simplicity of the IoT solution, and 2) clear incentives. Among the municipalities that have not embraced IoT, commonly cited barriers are economic factors and that other, more politically charged, issues take priority. This paper extends our understanding of public sector perception of IoT, as well as provides a comprehensive outlook on drivers for IoT-adoption.
\end{abstract}

\section{Introduction}

The Internet of Things (IoT) is a collective term for the implementation of sensor-based systems in everyday objects such as machines, vehicles, household appliances, clothes etcetera, and connecting them to the Internet. IoT does not represent a single technology, but rather a convergence of different streams in the development of hardware, software and sensory equipment that has been brewing for many years $[1,2,3]$.

One expected large area of application for IoT is within the smart cities discourse, contributing to everything from traffic flows and logistics [4], to street lighting [5], energy savings, and environment monitoring [6] through the use of smart, connected sensors and systems. The implementation and use of various IoT solutions have the potential to improve economic and political efficiency, which in turn enables social, cultural, and urban development [7].
However, there is a dearth of knowledge regarding how smart cities are formed as few studies to date have empirically and systematically investigated the reasoning behind IoT adoption in municipal organizations. Indeed, many of the highly cited papers are conceptual (see e.g. $[4,7,8,9,10]$ ) or focused on a single case in large metropolitan areas (e.g. [11, 12]). The purpose of our study is to better understand how IoT is adopted and diffused in municipal organizations at large. Furthermore, municipalities provide a suitable basis on which to assess the state of IoT in the public sector in general given their wide range of public services. Hence, our research question is: What factors and incentives facilitate the adoption and diffusion of IoT in municipal organizations?

In this paper, we present the results from a survey of IoT use in Swedish municipalities. Sweden is comprised of 290 municipalities that differ significantly in population from approximately 2500 to 950000 citizens. Municipalities in Sweden are responsible for a wide range of civic services, including day care, education, social services, elderly care, emergency services, water supply and sewerage, building permits, waste management and environmental protection. The aim of the survey was to investigate the proliferation of IoT as well as to gain insight into the domains that are most susceptible to connected devices. The survey was conducted by telephone with the head of the ITdepartment as the primary point of contact for each municipality. Given the variety of IoT in both domains of application [13] and technical diversity [14], the study applies an inclusive perspective on IoT in order to capture a comprehensive view on the current state of implemented IoT solutions in municipalities across Sweden. Results show that 57\% of Swedish municipalities use IoT in some form. Current applications are predominantly driven by how easy they are to adopt and how clearly their use solve current problems and challenges. Furthermore, most use of IoT is in the form of stove-pipe systems, i.e. systems that are not integrated with the overall IT 
infrastructure. This paper contributes to our understanding of municipal adoption - as well as public sector perception - of IoT by gathering results from municipalities that differ substantially in size, population and geographical location.

\section{Related research}

In this section we first give an overview of the technology and infrastructure necessary to enact the IoT. Then we shift our focus to adoption of new technologies in the public sector before we move on to diffusion of innovation.

\subsection{IoT technology and infrastructure}

The term Internet of Things is used as a collective term to encapsulate a wide variety of occurrences where components, devices, or systems communicate with one another without necessarily involving human intermediaries [15]. In a broad sense, the IoT marks a progress from neatly separated physical systems (e.g. products or tools) and digital systems (e.g. portable computers or back-office IT resources) to amalgamations that may be referred to as cyberphysical systems [13], intelligent systems [16] or simply "smart" products [1]. The common theme is a non-separable combination of physical hardware and digital software along with sensors, data storage and remote connectivity. IoT may be associated with a number of generic capabilities that can enable or improve processes across a range of contexts, including the ability to accurately and remotely monitor products, measure performance, control activities, automate and optimize tasks, and learn by analyzing patterns over time $[1,17]$.

The ability to connect and remotely monitor and/or control products or processes is by no means a novelty [18]. However, it is not until recent years that we have seen a sharp increase in IoT solutions. There are two main reasons for this. First, the ongoing trend towards mobile IT and embedded systems has yielded a booming market for smaller and cheaper hardware [19]. Hence, the costs associated with adding sensory components that can convert analogue events to digital signals and convey them to the surrounding environment are propelled ever downward, virtually removing the threshold for adding "smart" features to different products. Second, with the advent of platforms and application programming interfaces (APIs) that permit interoperability, we are rapidly moving from closed systems to open systems [20] that are expandable as long as one abides by an established set of rules and regulations.

Cheaper, more capable hardware and the means to integrate equipment from different manufacturers combine to move us closer to the key enabler for IoT: a ubiquitous, affordable IT-infrastructure that can absorb and overcome heterogeneity. Figure 1 provides an overview of the discrete layers of an IoT infrastructure.

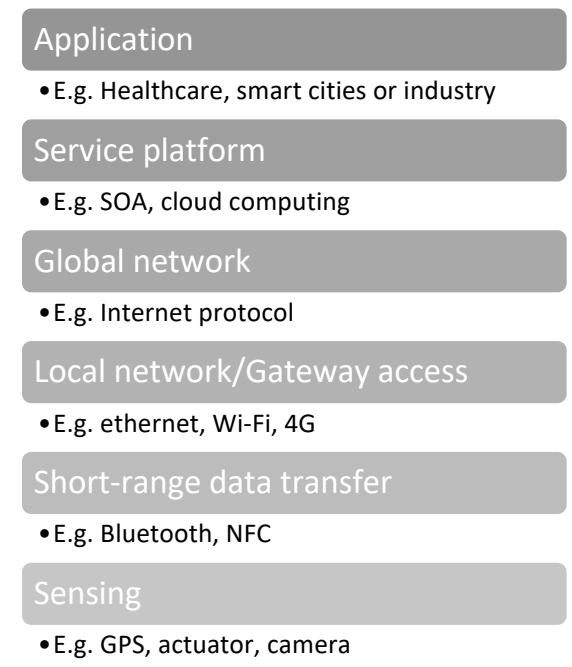

Figure 1. IoT infrastructure. [13]

\subsection{IoT in the public sector}

While still in its infancy, the application of IoT in the public sector can eventually bring about smart cities that benefit government as well as individual citizens. Borgia [13] identifies three major domains of IoT applications: industry, smart cities, and health $\&$ well-being. Public sector applications of IoT are predominantly found in the health $\&$ well-being and smart city domains. In relation to health \& wellbeing, IoT provides tools that enable relative independence for citizens who suffer from chronic conditions. Connected, user-friendly equipment can enable outpatients to monitor their own condition and transmit pertinent data to their doctor without needing to visit the hospital on a regular basis [21, 22]. In addition, IoT may also promote security for elderly or disabled citizens. For instance, connected equipment ranging from specialized pressure pads to generic smartphones may detect if someone has collapsed and needs assistance [23, 24].

IoT applications for health and well-being in the public sector may in many cases be counted as part of the smart cities' discourse, as many such solutions are implemented in order to improve the quality of 
life for citizens. Other potential application areas within the smart city domain include diverse areas like commuting \& mobility, adaptive electrical grids, "smart" buildings, environmental monitoring and public safety. Adaptive power grids and smart buildings can serve to reduce power consumption as well as mitigate the risk of brownouts during peak hours by distributing electrical usage more evenly across the course of the day [25]. Moreover, an interesting prospect is the idea of individual households producing electricity (e.g. via solar panels) and selling power back to the power grid [26], thus moving urban communities closer to a circular economy. IoT can also promote citizen safety by continuously monitoring urban environments and sending automated alerts in case of any deviations from the norm. The city of Chicago provides one of the more ambitious examples with their project Array of Things [11]. The aim of the project is to have some 500 "nodes" that provide constant updates on several variables, including temperature, humidity, air quality, and sound pollution on a block-by-block basis.

The application of IoT within the public sector can lead to more efficient and effective public services [12, 27], as well as provide better delivery of public services and support for citizen participation [28]. However, in order for the potential value of public sector IoT to be realized, IoT has to be not only adopted, but also widely diffused. Network density has been shown to have a positive effect on innovation spillovers and value creation [29], and the expectation is that once IoT takes hold and sensors are more widely distributed, more innovative solutions will follow, and greater values can be created. In the next section we take a closer look at the adoption and diffusion of innovation from an IoT perspective.

\subsection{Adoption and diffusion of innovation}

As the technical barriers to adopt IoT crumble, the range of available applications spans from expensive and tailored, to relatively cheap and innovative, where individual entrepreneurs or developers can act as part of a network or ecosystem rather than attempt to deliver a fully integrated system themselves [30]. However, while IoT carries with it an air of openness and ubiquity, novel solutions will still spread via established channels. The relevance, merit, and implementation of connected devices will vary considerably across different contexts, and in the public sector, where officials are elected for a limited term, subject to popular approval, and have short term budget- and planning horizons [31] the perceived potential for IoT value creation will determine the priorities of internal innovative processes. Rogers [32] describes four elements that determine how an innovation will disseminate.

First, the perceived attributes of the innovation itself is a fundamental determinant of its appeal in the eyes of prospective adopters. Rogers describes five key attributes: Relative advantage over existing approach, compatibility with existing values and needs, perceived complexity, trialability and opportunity to test the innovation, and finally observability of the innovation's results. The significance of the innovation itself is also highlighted in several models of IT adoption, notably TAM [33] and TAM2 [34] as well as perception of IT innovation [35].

Second, the channels through which proponents and potential adopters communicate are important as they determine how an innovation is presented and given meaning. Communication channels that promote interaction between individuals that share values, profession, background etc., tend to promote diffusion as the innovation can then be presented through the vernacular of a particular community.

Third, the social system in which an innovation diffuses, e.g. a town, organization or a community with a shared interest, is imbued with its own norms, structures and boundaries that determine how an innovation can diffuse. For instance, a particular innovation can diffuse quite rapidly within a given community but fail to propagate further if there are no bridges to other communities. Roger's second and third elements are echoed in the notions of subjective norms found in TAM2 [34] and social influence found in the Unified Theory of Acceptance and Use of Technology [36].

Fourth, time is a relevant factor to describe a decision unit's (e.g. a person or an organization) propensity to innovate. Rogers distinguishes between innovators, who actively seek out new ideas and take risks, early adopters, who are often considered opinion leaders in their respective social system, early and late majority, who proceed when it is advantageous (or necessary) and finally laggards, who are typically resistant to all change.

As the public sector is a large domain, the number of potential IoT solutions is tremendous. Different domains of IoT applications [13] carry with them different social systems and communication channels which in turn shape how innovations within each domain are perceived. While healthcare applications require extremely high reliability and low latency, RFID-tags used in logistics will likely prioritize low costs and low power consumption in order to tag and 
track large amounts of items over an extended period of time. In practice, industry boundaries, professional allegiances, or research communities can each form the basis for social systems that promote their own communication channels and thus aid an innovation's diffusion within a domain, but impede its progression onto a wider stage [37]. To better understand the diffusion of IoT within the public sector, this paper offers a systematic investigation of IoT usage in Swedish municipalities.

\section{Research method}

This paper features a survey of public sector use of IoT within Swedish municipalities. In line with interpretive methods of research within IS [38], our research aimed at providing an understanding of how IoT artifacts and sensor-based systems interact with their surroundings [39].

\subsection{Data collection}

The survey was initiated in December of 2016 with a pilot study of ten randomly selected Swedish municipalities. The results of the pilot led to minor refinements of the interview manuscript as well as the adoption of a new survey tool that supported collection and organization of data. A second pilot with twenty randomly selected municipalities was conducted in February-March of 2017. As both interview manuscript and survey tool proved satisfactory, we proceeded to gradually approach all 290 municipalities. We made at least three attempts on separate dates and times to contact each municipality. 87 out of 290 municipalities agreed to participate in our study, yielding a response rate of $30 \%$. The survey concluded in June 2017.

The municipalities differed in size from small to medium and large (see Table 1). We sought out ITmanagers in order to promote similar points of contact across all municipalities regardless of size. We assume IT-managers to be in a position to provide an overall perspective of IoT in the municipality - alternatively provide us with contact information to a more suitable point of contact.

\begin{tabular}{|l|l|l|}
\hline Size & No.of citizens & $\begin{array}{l}\text { Municipalities in Sweden } \\
\text { (No. in this study) }\end{array}$ \\
\hline Small & $<15000$ & $132(44)$ \\
\hline Medium & $15000-50000$ & $110(29)$ \\
\hline Large & $>50000$ & $48(14)$ \\
\hline
\end{tabular}

Table 1. Size and number of municipalities

Data was primarily collected via structured interviews performed via telephone. Interviews were recorded and lasted for an average of 20 minutes. Prior to initiating the survey, researchers conducted an initial review of municipal websites. The review revealed that websites were strikingly homogeneous in their division of municipal responsibilities into a number of areas: Home care \& support, education \& childcare, traffic \& infrastructure, construction, living $\&$ environment, and culture \& leisure. Assuming that respondents would be familiar with this allocation of function and responsibility, we adopted this division as themes for our survey and asked the respondents for examples of IoT implementations within each respective area. For each instance of IoT implementation, the respondent was also asked when it had been adopted, its primary users, why this particular solution had been adopted, who was the developer of the solution and whether or not it lived up to expectations. Respondents were encouraged to provide descriptive, exhaustive answers. In cases where the municipality did not offer any applications of IoT, we instead posed questions regarding their perspective on connected devices and initiatives pertaining to digitalization of municipal activities and services. Additionally, all interviews included introductory questions regarding the municipality's digital strategy and whether IoT was part of their agenda.

On two occasions, the respondent declined to participate in a phone interview, but agreed to answer questions sent via e-mail. These responses were also fed into the survey tool.

\subsection{Data analysis}

The data analysis was conducted in three steps. First, we used the survey tool to provide a summary of the response rate and other key factors listed under Section 4. Second, we scrutinized responses to see which types of IoT application were prevalent and in which areas they were applied. In keeping with an interpretive approach [40], we reviewed the responses from each municipality manually and summarized the nature of the IoT application used and circumstances surrounding its adoption in search of common themes as well as notable deviations. In some cases, we went back to the original recording of the interview in order to ensure that we did not miss any significant detail. Having manually reviewed the data, the authors discussed the findings with a particular emphasis on recurring themes in order to minimize subjective bias and promote shared, intersubjective interpretations of the results [41]. Third, we applied our findings to answer our research question pertaining to the factors and incentives that facilitate the adoption and diffusion of IoT in 
municipal organizations. In the following sections, we present our results together with some direct quotes from the interviews and discuss the implications of our research.

\section{Results}

Participating municipalities exhibit a wide distribution in terms of size as well as geographical location, indicating that the study captures a representative cross section of current views and priorities.

Our study shows that larger municipalities, as well as those who have a working relationship with external parties are more likely to adopt IoT. These municipalities are aware of the benefits of connected solutions and they typically use IoT in some form already. However, they also emphasize the need for a long-term strategy for integrating a variety of technical solutions. "We look at many cases where we use sensors, everything from lamp posts to sensor systems, in order to make smarter and better planned decisions. And then we look at another level, it's about aggregating data: we systematize and analyze machine-readable data, which I think is more important than the actual sensor question: "How do we use the data?" (Municipality 20/Large). The overall purpose of such a strategy is to manage the huge volumes of data generated by connected devices as well as formulate workable standards for procurement processes so that any new product or component fits with the overall infrastructure. Security and integrity are considered important issues that remain a challenge that needs to be fully resolved before they commit any major investments to IoT.

Other municipalities consider IoT relevant to a certain degree. They do in some cases offer examples of technical solutions, e.g. based on sensors that could fall within the scope of IoT, but the respondents see their application as an example of digitalization or modernization of municipal tools and services. Some municipalities describe a strong interest in IoT within certain administrative departments where new tools offer tangible value. These discussions tend to take place at an operative rather than strategic level and are motivated by practical benefits and solutions to everyday problems.

The municipalities that do not currently consider IoT a relevant issue state that they do not see any direct benefits with IoT or that adopting IoT would involve excessive effort or financial re-sources. The same municipalities concede that IT generally leads to improvements in efficiency, such as e-services for citizens, and qualitative improvements, such as access to computers in schools. However, the adoption of IoT would demand skills and resources that the municipality is currently unable or unwilling to commit. "There has to be a change in all areas of operation, in the way people think [...]. This is a big challenge: To raise the general level of computer maturity and understanding of security issues and such things. We talk a lot about that now" (Municipality 21/Small).

Figure 2 shows the prevalence of IoT-applications among the most commonly specified areas. It is readily apparent that two areas, home care \& support, and construction, living \& environment, are overrepresented among the examples provided during the course of our survey. We will now provide a more detailed account of each area.

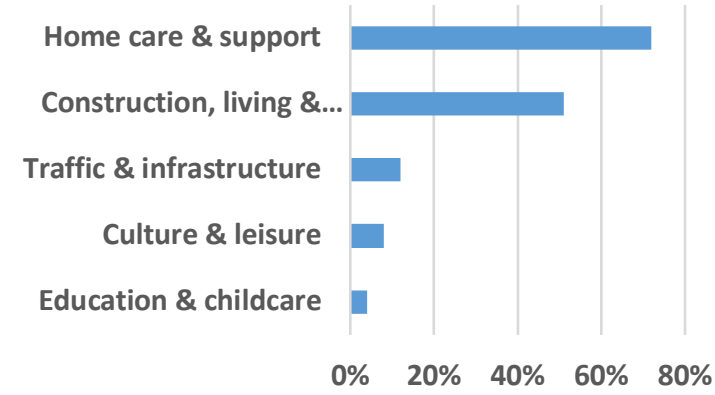

Figure 2. Prevalence of IoT applications.

\subsection{Home care \& support}

The area of home care \& support covers all manner of activities managed by social services. One of their chief responsibilities is outpatient care of citizens who require day-to-day assistance but are not under active medical care at any hospital. The most commonly cited activities in our study were care for senior citizens that reside in private residences rather than dedicated nursing homes or retirement facilities.

Keyless locks consist of a small box mounted inside the front door of a residence. The box houses a mechanism that turns the (existing) physical lock in order to open the door. The purpose of keyless locks is to remove the requirement for nursing staff to carry physical keys for every individual apartment they visit during their shift. Instead, the keyless locks may be opened using an app on a smartphone. In addition to nursing staff, keyless locks are also useful to other healthcare personnel who are able to respond to emergency situations without first securing access to a physical key. Hence, this solution can significantly shorten the response time in case of medical emergencies.

Night-time attendance is performed by nursing staff in cases where there is a need to attend to the 
health and safety of a person during the night, e.g. due to a physical handicap or dementia. This is normally conducted via visits to the care recipients' homes at scheduled intervals during the night. Digital night-time attendance means that the check-in is performed by means of a camera rather than a physical visit. Cameras may only be used at specific intervals during the night and thus cannot be used arbitrarily by care providers. Digital night-time attendance offers advantages to care recipients, who may be disturbed by people entering their home during the night, as well as to nursing staff, who can spend more time with those who really need help rather than organize their shifts around a fixed schedule. "I think it is marketed with too much focus on the technology. They call it camera surveillance; I'd like to think of it as a non-disruptive disturbance" (Municipality 8/Large).

Pressure-sensitive floor pads vary in size and configuration but are placed on surfaces where care recipients spend a lot of time or are at increased risk of falling, e.g. by the bed or in the bathroom. Sensors in a floor pad can, based on distribution of weights or force of impact, detect if a person has fallen over and alert nursing staff of the situation. Hence, caregivers are able to render aid even if a person is unconscious or otherwise unable to summon assistance himself or herself.

\subsection{Construction, living $\&$ environment}

Construction, living and environment is, alongside home care $\&$ support, an area that enjoys a relatively high diffusion of IoT applications. The area includes building construction and maintenance, municipal services in relation to housing, and waste management and sewage treatment. The municipalities featured in our study have implemented IoT applications related to waste management, ventilation systems, and flow monitoring in water treatment plants. The main reason for adoption is efficiency and increased control, but there is also an awareness of the increased risk of hacker attacks as connectivity increases: "There are of course a lot of different things that can be monitored and controlled in a better way, but at the same time we know that there are certain security risks associated with this" (Municipality 37/Medium).

Several municipalities have equipped their garbage trucks with GPS transponders and a tablet computer that provides the driver with the planned route. Furthermore, curb side garbage containers (which are owned by the municipality and distributed to individual households) are equipped with RFID tags that are automatically scanned as the containers are emptied, ensuring that no container is missed, and permitting individual billing of households based on the amount of garbage they produce.

Connected ventilation systems offer the possibility to monitor, and to some extent remotely control, municipal buildings. If some part of the ventilation system ceases to function or displays aberrant behavior, an alarm is sent (e.g. by text message) to a service technician. With advance information, technicians can often fix the problem on the first visit rather than having to do multiple trips in order to get the right tools or spare parts. Furthermore, the information system can be used for historical data so that technicians can see if any particular error has arisen on multiple occasions and, if needed, investigate underlying problems.

The possibility to remotely monitor systems and devices is also relevant in relation to pumping stations, water treatment plants and other facilities where the municipality has an obligation to ensure compliance with water quality standards. By installing sensors that continuously monitor water quality, staff do not have to waste time travelling to each installation and conducting manual inspections.

\subsection{Traffic \& infrastructure}

Traffic and Infrastructure includes activities relating to the monitoring and/or control of city traffic, parking spaces as well as maintaining roads and street lights.

A few municipalities stated that they use sensors to monitor traffic flow in the city and have started testing similar technology to help motorists find parking spaces as well as adapt street lighting in different areas based on the level of activity. These applications are primarily applied by larger municipalities that see a need to monitor traffic flows along major traffic routes and quickly respond to accidents or emergencies. "We want to use IoT as a decision support system, to see how the traffic works with traffic lights, traffic control, buses, public transportation, etc. in order to offer better services to our citizens" (Municipality 14/Large).

In addition, larger municipalities see a clear need to promote public transportation in order to reduce the number of cars on the roads. Here, IoT solutions can play a role where a connected bus or train can provide data on traffic conditions or delays across the city. This information then provides a basis for digital services that can alert commuters in case of delays and suggest alternate routes. 


\subsection{Culture \& leisure}

The area of culture and leisure includes municipal activities related to libraries, arts, theater and tourism.

Our study provided a few examples of IoT applications in this area, primarily related to libraries and cultural centers. By integrating new technology in the premises, municipalities hope to offer new opportunities to engage the visitor, e.g. through augmented reality where the physical space is in some way changed or improved via a smartphone app or VR headsets. Another use of IoT was the use of RFID tags to enter the public swimming pool and sensors to control water temperature and purification among other relevant factor. For some, the possibilities seemed endless: "Once you start implementing these things [IoT], it grows. You don't even realize how much potential it holds" (Municipality 81/Medium). In addition, IoT was used to tag books in libraries to ensure their return to the right shelf, and to estimate average time spent queueing, e.g. in amusement parks.

\subsection{Education \& childcare}

The area of education \& childcare includes education from preschool up to secondary school as well as municipal adult education. It also includes leisure activities organized by municipalities.

Only two out of 87 participating municipalities indicated that they use some form of IoT solution in this area. In both cases, it is a system used to register attendance for children attending preschool. When arriving at their classroom, children register attendance by either touching their portrait on an interactive screen or by swiping a RFID-tag (embedded in a durable plastic casing) in front of a sensor. The system allows teachers quick and easy overview of attendance so that they know if anyone is missing - a feature that can be particularly valuable for substitute teachers. A smartphone app gives parents real-time updates on when their child has checked in as well as checked out from school.

\subsection{Summary of results}

The overall motives for municipal adoption of IoT can be expressed in terms of a clear and present necessity to be more effective in managing municipal resources: time, money and staff. "The primary reason [for IoT adoption] is to create a better work environment for our staff, but also of course to provide better services to citizens" (Municipality 44/Small) Among the municipalities that do not feature any application of IoT, economic factors were often cited as the main barrier. In the face of budgetary constraints, issues such as the introduction of digital tools in primary school are given priority over IoT investments.

Experience from the requirements-driven solutions in use today also provides a clear indication of the potential for new technical solutions and its relevance to long-term strategic development. Many municipalities seek support and guidance on how to integrate the functionality and opportunities brought by IoT into future procurement contracts with external suppliers. "After all it is really important when you are in the procurement stage, that you do it in a good way. [...] The development is quite fast in this area... there are a number of suppliers who deliver this stuff, and you are quite dependent on what the market can offer" (Municipality 43/Small). Two areas; home care \& support, and construction, living \& environment, stand out in our survey as very well disposed to IoT-applications. All municipalities have a responsibility towards their citizens to provide care for elderly or disabled citizens as well as ensure access to necessities such as clean water and electricity. A contributing factor to the prevalence of IoT in these areas is the availability of mature and cost-effective solutions that are attractive to municipalities where budgetary constraints and financial justification are constantly recurring issues in all purchases and investments.

In addition, many of the applications of IoT found in these areas were initiated and implemented by the respective administrative department entirely without the knowledge or involvement of the IT department. This suggests that IoT adoption is not primarily driven by concerted strategic efforts, but rather individual departments or professional groups that see a potential to resolve bottlenecks in their processes or improve the quality of service.

\section{Discussion}

Even though all municipalities have similar duties and commitments to their citizens, one cannot ignore the fact that they face different conditions. Their unique situations inevitably affect their perspective on new technologies as a solution to their challenges. In rural areas, people often have to travel long distances for public services, which means that even small, fiscally conservative municipalities see great value in replacing manual intervention on-site with automation or remote manual intervention where possible. Metropolitan areas also share a general desire to utilize their resources as efficiently as possible. However, they also approach IoT as an 
absolute necessity to ensure long-term supply of electricity, potable water, public transportation, traffic management and social services for an everincreasing population. The IoT-applications that see the widest diffusion are characterized by two properties: simplicity and clear incentives. In the vernacular of Rogers [32], simplicity may be expressed in terms of complexity and trialability of the innovation, whereas incentives relate primarily to relative advantage and observability.

The IoT represents a wide diversity of technologies [14] that combine into a multi-layered hierarchy [13] where data are captured, transmitted, aggregated, analyzed and finally presented to the user. The level of complexity is daunting even to those favourably inclined to new technologies. This study shows that relatively mature "off-the-shelf" IoT- solutions promote simplicity, as they reduce the user-level complexity. Moreover, these solutions represent vertically integrated applications that stand separate from other municipal systems. Hence, they lend themselves to non-committal trial periods where users have an opportunity to test the new technology and gradually learn to incorporate the new tool into their daily routine.

Furthermore, the majority of IoT-applications reported in our study are associated with clear incentive structures. The applications of IoT found in home care \& support as well as construction, living $\&$ environment both offer relative advantages and yield observable results. Both characteristics coincide in the ability to economize on resources by introducing new technical solutions. As such, our study provides empirical support for the conceptual benefits presented in extant research on public sector IoT (e.g. [4, 9, 10]). However, we find it more important to note that cost saving IoT is often implemented as a quality enhancing measure. That is, IoT applications enable a better match between operational requirements and the availability of human resources. Personal alarms, pressure sensitive pads and similar "smart" technologies permit social services to better allocate their staff and spend more time with citizens in actual need of assistance. The same logic is applicable to monitoring municipal sewage treatment plants and similar installations, where maintenance staff can continuously ensure water quality instead of relying on intermittent manual measurements. Furthermore, automated monitoring can be linked to alarms that alert technicians as soon as any deviation from acceptable values is detected.

Looking beyond the nature of the innovation itself, our study revealed that IoT was primarily adopted on an operational rather than strategic level, where individual departments selected, tested and implemented different IoT applications. Hence, it is the individual departments and their respective professionals rather than municipalities as a governmental organization that drive IoT adoption. Rogers offers a plausible explanation as he argues that the communication channels and social systems through which news on an innovation propagates are both core factors of innovation diffusion. Our study suggests that the most salient social system with regards to adoption of IoT is not the municipality, but rather the profession. Extant research argues that a key barrier to innovation in the public sector is the disconnect between advocating for an innovation which usually happens in city councils or similar upper management levels - and realizing an innovation - which happens at middle management and operational levels [42]. The disconnect between policy and implementation usually brings about stress as well as a perceived necessity to please policy makers rather than the intended beneficiaries, i.e. citizens. Communications channels between established professionals not only promote familiarity and credibility, but also remove the sense of disparity in power and influence.

The study does not offer any straightforward results in relation to the fourth element of diffusion time. Indeed, while Rogers describes five homogeneous categories of adopters, our study shows that adoption of IoT is distinctly heterogeneous and dependent upon - rather than separate from - the other elements of diffusion. That is, the propensity of a municipality to adopt a given innovation is influenced by 1) the availability of mature, easily understood applications and 2) the willingness of different professional groups to accept new technologies and tools. Hence, the same municipality may be part of the early adopters or early majority in one area of application, and yet a laggard in another depending on the relative interest and influence of different professional groups. Furthermore, the uptake of IoT was greater among larger municipalities as well as those with close ties to colleges or universities, suggesting that ample access to resources, either in the form of tax revenues or knowledge, may both be beneficial to the diffusion of innovation.

Finally, our study also identified non-adopters. Their main reasons for not investing in IoT were mainly expressed as financial concerns, but their reasoning was often rooted in unfamiliarity with the technology. Given budget constraints, they were more likely to invest in something known, that did not require additional skillsets, new resources, or large educational efforts. This type of reasoning is 
not uncommon within the public sector, where officials are elected for a limited term, subject to popular approval, and have short term budget- and planning horizons [31]. Rather than creating shared, long-term strategies there is a tendency to invest in technology based on operational needs from individual departments. This in turn has an effect on the overall diffusion of IoT in the public sector and is something that municipalities need to address in order for IoT to progress onto a wider stage.

\section{Conclusions}

Out of 87 participating municipalities, 50 responded that they today use some form of IoT application. If this proportion is valid for Sweden as a whole, it means that over $57 \%$ make use of IoT in some form. The study shows that two areas are overrepresented among the applications used today: home care \& support as well as construction, living \& environment. We can discern two distinctive factors that characterize IoT applications within these areas: clear incentives and simplicity.

The obligation to provide a high level of service drives the adoption of new technologies and new solutions. In-home care and elderly care are clear examples of activities in need of new solutions that can contribute to greater efficiency without impairing quality of care or work environment.

The areas of home care \& support as well as construction, living \& environment both feature a number of off-the-shelf solutions available on the market. Ventilation equipment and water purification systems offer remote connectivity using different industry standards that require little to no effort to utilize. Similar phenomena are evident in social services and healthcare, where vendors offer various connected solutions that are easy to install and manage in either private residences or nursing homes.

While simplicity and clear incentives drive adoption of particular applications, our study shows that overall diffusion of IoT in each individual municipality is low and determined by operational needs from professional groups rather than long-term managerial strategies. Hence, we see a need for future research into drivers of comprehensive IoT adoption in public sector organizations.

Our findings offer limited generalizability as it is situated in a specific country. Another limitation of our study is the choice of IT manager as the primary point of contact. Our study shows that IT managers often have a good birds-eye perspective of municipal IT-systems but are less able to provide detailed insights into the respective applications. Future studies could either focus on a more operative level, in order to gain deep insights into specific solutions, or concentrate on a higher strategic level, that sets the agenda for the entire municipality.

\section{References}

[1] M.E. Porter, and J.E. Heppelmann, "How smart, connected products are transforming competition", Harvard Business Review, Vol. 92, No. 11, 2014, pp. 11-64.

[2] T. Saarikko, U. H. Westergren, and T. Blomquist, "The Internet of Things: Are you ready for what's coming?", Business Horizons, Vol. 60, No. 5, 2017, pp. 667-676.

[3] U. H. Westergren, T. Saarikko, and T. Blomquist, "Initiating the Internet of Things: Early Adopters' Expectations for Changing Business Practices and Implications for Working Life", in The Internet of People, Things and Services, C. Simmers and M. Anandarajan, Eds., Routledge, London, 2018, pp. 111131.

[4] P. Neirotti, A. de Marco, A. C. Cagliano, G. Mangano, and F. Scorrano, "Current trends in Smart City initiatives: Some stylised facts", Cities, Vol. 38, 2014, pp. 25-36.

[5] F. Leccese, M. Cagnetti, and D. Trinca, "A smart city application: A fully controlled street lighting isle based on Raspberry-Pi card, a ZigBee sensor network and WiMAX", Sensors, Vol. 14 No. 12, 2014, pp. 24408-24424.

[6] H. Schaffers, N. Komninos, M. Pallot, B. Trousse, M. Nilsson, and A. Oliveira, "Smart cities and the future internet: Towards cooperation frameworks for open innovation", in The future internet assembly, Springer, Berlin, Heidelberg, 2011, pp. 431-446.

[7] R. G. Hollands, "Will the real smart city please stand up? Intelligent, progressive or entrepreneurial?", City, Vol. 12, No. 3, 2008, pp. 303-320.

[8] T. Nam, and T. A. Pardo, "Conceptualizing smart city with dimensions of technology, people, and institutions", in Proceedings of the 12th annual international digital government research conference: digital government innovation in challenging times, ACM, 2011, pp. 282-291.

[9] K. Su, J. Li, and H. Fu, "Smart city and the applications", In International Conference on Electronics, Communications and Control (ICECC), IEEE, 2011, pp. 1028-1031.

[10] J. Jin, J. Gubbi, S. Marusic, and M. Palaniswami, "An information framework for creating a smart city through internet of things", IEEE Internet of Things Journal, Vol. 1, No. 2, 2014, pp. 112-121.

[11] C. E. Catlett, P. H. Beckman, R. Sankaran, and K. K. Galvin, "Array of things: a scientific research instrument in the public way", in Proceedings of the 2nd International Workshop on Science of Smart City Operations and Platforms Engineering, ACM, 2017, pp. 26-33.

[12] Y. M. Kim, H. S. Kim, S. Y. Moon, and S-Y. Bae, "Ubiquitous Eco-City Planning in Korea. A Project for 
the Realization of Ecological City Planning and Ubiquitous Network Society", in Proceedings of the REAL CORP 2009, M. Schrenk, V. Popovich, D. Engelke, and P. Elisei, Eds., 2009, REAL CORP, Spain, pp. 925-930.

[13] E. Borgia, "The Internet of Things vision: Key features, applications and open issues", Computer Communications, Vol. 54, 2014, pp. 1-31.

[14] L. Atzori, L, A. Iera, and G. Morabito, "The Internet of Things: A survey", Computer Networks, Vol. 54, No. 15,2010 , pp. 2787-2805.

[15] F. Wortmann, and K. Flüchter, "Internet of Things", Business \& Information Systems Engineering, Vol. 57, No. 3, 2015, pp. 221-224.

[16] M. Del Giudice, "Discovering the Internet of Things (IoT) within the business process management: A literature review on technological revitalization", Business Process Management Journal, Vol. 22, No. 2, 2016, pp. 263-270.

[17] M. Ferretti, and F. Schiavone, "Internet of Things and business processes redesign in seaports: The case of Hamburg", Business Process Management Journal, Vol. 22, No. 2, 2016, pp. 271-284.

[18] N. V. Wünderlich, K. Heinonen, A. L. Ostrom, L. Patricio, R. Sousa, C. Voss, and J. G. Lemmink, "“Futurizing" smart service: implications for service researchers and managers", Journal of Services Marketing, Vol. 29, No. 6/7, 2015, pp. 442-447.

[19] E. Brynjolfsson, and A. McAfee, The second machine age: Work, progress, and prosperity in a time of brilliant technologies, WW Norton \& Company, New York, 2014

[20] J. A. Stankovic, "Research directions for the internet of things", IEEE Internet of Things Journal, Vol. 1, No. 1, 2014, pp. 3-9.

[21] F. Delmastro, "Pervasive communications in healthcare", Computer Communications, Vol. 35, No. 11, 2012, pp. 1284-1295.

[22] Z. Pang, L. Zheng, J. Tian, S. Kao-Walter, E. Dubrova, and Q. Chen, "Design of a terminal solution for integration of in-home health care devices and services towards the Internet-of-Things", Enterprise Information Systems, Vol. 9, No. 1, 2015, pp. 86-116.

[23] S. Abbate, M. Avvenuti, F. Bonatesta, G. Cola, P. Corsini, and A. Vecchio, "A smartphone-based fall detection system". Pervasive and Mobile Computing, Vol. 8 No. 6, 2012, pp. 883-899.

[24] F. De Backere, F. Ongenae, F. van den Abeele, J. Nelis, P. Bonte, E. Clement, ... and F. de Turck, "Towards a social and context-aware multi-sensor fall detection and risk assessment platform", Computers in biology and medicine, Vol. 64, 2015, pp. 307-320.

[25] I. Colak, S. Sagiroglu, G. Fulli, M. Yesilbudak, and C. F. Covrig, "A survey on the critical issues in smart grid technologies", Renewable and Sustainable Energy Reviews, Vol. 54, 2016, pp. 396-405.

[26] E. Ancillotti, R. Bruno, and M. Conti, "The role of communication systems in smart grids: Architectures, technical solutions and research challenges", Computer Communications, Vol. 36, No. 17-18, 2013, pp. 1665-1697.
[27] C. Balakrishna, "Enabling Technologies for Smart City Services and Applications", in Proceedings of IEEE International Conference on Next Generation Mobile Applications, Services and Technologies, IEEE, Paris, France, 2012, pp. 223-227.

[28] S. Allwinkle, and P. Cruickshank, "Creating Smarter Cities: An Overview", Journal of Urban Technology, Vol. 18, No. 2, 2011, pp. 1-16.

[29] K. Meagher, and M. Rogers, "Network density and R\&D spillovers", Journal of Economic Behavior \& Organization, Vol. 53, No. 2, 2004, pp. 237-260.

[30] K. Prince, M. Barrett, and E. Oborn, "Dialogical strategies for orchestrating strategic innovation networks: The case of the Internet of Things", Information and Organization, Vol. 24, No. 2, 2014, pp. 106-127.

[31] D. Grant, "Barriers to Public Sector Innovation", Doctoral dissertation, Northumbria University, Newcastle upon Tyne, 2016.

[32] E. M. Rogers, "Diffusion of innovations", Simon and Schuster, New York, $5^{\text {th }}$ ed., 2003.

[33] F. D. Davis, "Perceived usefulness, perceived ease of use, and user acceptance of information technology", MIS Quarterly, Vol. 13, No. 3, 1989, pp. 319-340.

[34] V. Venkatesh, and F. D. Davis, "A theoretical extension of the technology acceptance model: Four longitudinal field studies", Management Science, Vol. 46, No. 2, 2000, pp. 186-204.

[35] G. C. Moore, and I. Benbasat, "Development of an instrument to measure the perceptions of adopting an information technology innovation", Information Systems Research, Vol. 2, No. 3, 1991, pp. 192-222.

[36] V. Venkatesh, M. G. Morris, G. B Davis, and F. D. Davis, "User acceptance of information technology: Toward a unified view", MIS Quarterly, Vol. 27, No. 3, 2003, pp. 425-478.

[37] P. DiMaggio, and W. W. Powell, "The iron cage revisited: Collective rationality and institutional isomorphism in organizational fields", American Sociological Review, Vol. 48, No. 2, 1983, pp. 147160.

[38] G. Walsham, "Interpretive case studies in IS research: nature and method", European Journal of Information Systems, Vol. 4, No. 2, 1995, pp. 74-81.

[39] H. K. Klein, and M. D. Myers, "A set of principles for conducting and evaluating interpretive field studies in information systems", MIS Quarterly, Vol. 23, No. 1, 1999, pp. 67-93.

[40] G. Walsham, "Doing interpretive research", European Journal of Information Systems, Vol. 15, No. 3, 2006, pp. $320-330$.

[41] W. J. Orlikowski, and J. J. Baroudi, "Studying information technology in organizations: Research approaches and assumptions", Information Systems Research, Vol. 2, No. 1, 1991, pp. 1-28.

[42] J. Potts, and T. Kastelle, "Public sector innovation research: What's next?", Innovation, Vol. 12, No. 2, 2010, pp. 122-137. 